\title{
INTERMEDIATE-TERM OUTCOME OF MITRAL RECONSTRUCTION IN CARDIOMYOPATHY
}

Steven F. Bolling, MD

Francis D. Pagani, MD, PhD

G. Michael Deeb, MD

David S. Bach, MD
Objective: Severe mitral regurgitation is a frequent complication of endstage cardiomyopathy that contributes to heart failure and predicts a poor survival. We studied the intermediate-term outcome of mitral reconstruction in 48 patients who had cardiomyopathy with severe mitral regurgitation and were operated on between June 1993 and June 1997. Methods: Ages ranged from 33 to 79 years $(63 \pm 6$ years) with left ventricular ejection fractions of $8 \%$ to $25 \%(16 \% \pm 3 \%)$. All patients were receiving maximal drug therapy and were in New York Heart Association class III-IV with severe, refractory 4+ mitral regurgitation. Operatively, all 48 had undersized flexible annuloplasty rings inserted, 7 had coronary bypass grafts for incidental disease, 11 had prior bypass grafts, and 11 also had tricuspid valve repair. Results: One operative death occurred as a result of right ventricular failure. Postoperative transesophageal echocardiography revealed mild mitral regurgitation in 7 patients and no mitral regurgitation in 41 . There were 10 late deaths, 2 to 47 months after mitral reconstruction. The 1- and 2-year actuarial survivals have been $82 \%$ and $71 \%$. At a mean follow-up of 22 months, the number of hospitalizations for heart failure has decreased, and 1 patient has had heart transplantation. Significantly, New York Heart Association class improved from 3.9 \pm 0.3 before the operation to $2.0 \pm 0.6$ after the operation. Twenty-four months after the operation, left ventricular volume and sphericity have decreased, whereas ejection fraction and cardiac output have increased. Conclusion: Whether this favorable modification of left ventricular function and geometry will persist remains unknown. However, mitral repair for cardiomyopathy with mitral regurgitation allows new strategies for these patients. (J Thorac Cardiovasc Surg 1998;115:381-8)
$H$ parti eart transplantation is standard treatment for patients with severe congestive heart failure associated with end-stage heart disease. Although successful, transplantation is hindered by donor shortage and its limited applicability to older patients or those with comorbid diseases. As a result, waiting times and recipient mortality on the waiting

From the Sections of Thoracic Surgery and Cardiology, The University of Michigan, Ann Arbor, Mich.

Read at the Twenty-third Annual Meeting of The Western Thoracic Surgical Association, Napa, Calif., June 25-28, 1997.

Received for publication July 8, 1997; revisions requested August 27, 1997; revisions received Oct. 9, 1997; accepted for publication Oct. 9, 1997.

Address for reprints: Steven F. Bolling, MD, The University of Michigan Hospitals, Section of Thoracic Surgery, 2120D Taubman Center, Box 0344, Ann Arbor, MI 48109.

Copyright (C) 1998 by Mosby, Inc.

$0022-5223 / 98 \$ 5.00+0 \quad \mathbf{1 2 / 6 / 8 6 8 8 4}$ list have increased. New surgical strategies to manage severe end-stage heart disease have therefore evolved, including coronary artery revascularization, ${ }^{1}$ cardiomyoplasty, ${ }^{2}$ left ventricular (LV) myoreduction surgery, ${ }^{3}$ and mitral valvular repair. ${ }^{4-7}$ Mitral regurgitation (MR) is a significant complication of end-stage cardiomyopathy, resulting from dilation of the annular-ventricular apparatus, ${ }^{8}$ altered ventricular geometry, ${ }^{9}$ or papillary muscle dysfunction. ${ }^{10}$ MR exacerbates the volume overload of the already dilated left ventricle, with progressive annular dilation, worsened MR, and increased heart failure, and it predicts a poor survival. ${ }^{11}$ Encouraged by a prior study that assessed the feasibility of mitral reconstruction in dilated cardiomyopathy, we undertook this study to examine the intermediate outcome of mitral reconstruction in terms of patient survival and impact on heart failure and LV function, geometry, and remodeling. 


\section{Methods}

Between June 1993 and June 1997, 48 consecutive, nonrandomized patients at the University of Michigan Medical Center, with end-stage dilated cardiomyopathy and refractory severe MR, were studied prospectively. All patients had New York Heart Association (NYHA) class III or IV congestive heart failure despite receiving maximized medical regimens and had severe LV systolic dysfunction defined as an ejection fraction less than $25 \%$ on angiography or radionuclide studies. Patients were excluded from this study if there was evidence of primary mitral valvular disease, if significant MR preceded cardiomyopathy, if surgical intervention included revascularization of viable myocardium, or if there was significant aortic insufficiency.

MR was assessed by means of color-flow Doppler ultrasonography; severity was graded as mild, moderate, or severe. Quantitative analysis was performed by a single experienced echocardiographer blinded to clinical data. Measurements of LV, mitral annular, and outflow tract diameters were made from two-dimensional images. LV volumes, stroke volume, flows, regurgitant fractions, and ejection fraction were calculated as previously described. ${ }^{4}$ Intraoperative and postoperative mitral gradients were calculated by means of continuous wave Doppler ultrasonography.

Mitral valve reconstruction was performed through a median sternotomy with standard cardiopulmonary bypass and hypothermic blood cardioplegic arrest in 37 patients and through a right thoracotomy with cold fibrillatory arrest in 11 patients who had prior coronary bypass grafting. Operations were performed with institutional approval and informed consent.

All patients were contacted by telephone and personal office visit for follow-up. A standardized questionnaire was used for symptomatic evaluation, with physical activity being quantitated by self-report and compared with preoperative functional status. The patients' personal physicians were also contacted in follow-up. Echocardiographic follow-up studies were generally performed at the University of Michigan. In the case of echocardiograms performed elsewhere, original videotapes were sent for quantitative analysis at the University of Michigan. All data are presented as mean \pm standard deviation. Comparisons between preoperative and postoperative maximum volume of oxygen use, NYHA class, LV size, volume, ejection fraction, regurgitant fraction, and forward cardiac output were made by means of paired Student's $t$ tests. Survival analysis was performed by the Kaplan-Meier method. A significance level of 5\% was used when comparing differences.

\section{Results}

Forty-eight patients (31 men) were included in the study. Their ages ranged from 33 to 79 years (mean $63 \pm 6$ years). Twenty patients were over the age of 65 years. Twenty-four patients had nonischemic dilated cardiomyopathy, and 24 had end-stage ischemic cardiomyopathy, without ongoing ischemia, as defined by negative dobutamine echocar- diographic functional testing and/or negative positron emission tomography scanning for viability. No patient was believed to have an acute myopathy. Preoperatively, 40 patients had NYHA class IV congestive heart failure and eight had class III. The mean duration of documented cardiomyopathy or symptomatic congestive heart failure was 46 years (range 0 to 16 years). In the year before the operation, these patients had up to nine hospital admissions for congestive heart failure. All patients were receiving maximized medical therapy for congestive heart failure including digoxin, diuretics, and afterload reducers. Afterload reduction was accomplished with angiotensin-converting enzyme inhibitors in all patients, with the addition of either nitroglycerin or hydralazine, as indicated.

Preoperative ejection fraction ranged from $8 \%$ to $25 \%$ (mean $16 \% \pm 3 \%$ ). On coronary angiography, seven patients with nonischemic dilated cardiomyopathy were found to have incidental coronary artery disease. All patients with ischemic cardiomyopathy had significant coronary artery disease, with prior coronary artery bypassing, but no ongoing ischemia on functional testing. One patient with ischemic cardiomyopathy had a history of recurrent ventricular tachycardia. Another patient, with nonischemic cardiomyopathy, had hypereosinophilia syndrome and was receiving interferon therapy. This patient was not believed to be a candidate for transplantation.

All patients underwent mitral reconstruction by means of a remodeling ring annuloplasty, with implantation of an undersized flexible remodeling ring. No patient required a complex repair, inasmuch as all patients were believed to have only annular dilation or papillary muscle dysfunction (or both). The average duration of cardiopulmonary bypass was $124 \pm 24$ minutes (range 66 to 198 minutes), with an aortic crossclamp time of $71 \pm 11$ minutes (range 51 to 123 minutes). None of the patients entered in this study had irreparable heart disease. Coronary artery bypass grafts were placed in seven patients with incidental coronary artery disease. Additional procedures included De Vega tricuspid annuloplasty in 11 patients, closure of an atrial septal defect and a patent foramen ovale in 12 , and placement of implantable cardioverter-defibrillator patches in one patient.

One operative death from right ventricular failure occurred in a patient who died despite use of both an intraaortic balloon pump and mechanical support with a right ventricular assist device. All other 
patients survived the mitral reconstruction procedure, despite their poor preoperative ventricular function. All patients were weaned from cardiopulmonary bypass with the use of a phosphodiesterase inhibitor and norepinephrine. One patient required support with an intraaortic balloon pump for 2 days. Intraoperative transesophageal echocardiography after annuloplasty and the discontinuation of cardiopulmonary bypass revealed no MR in 41 patients and trivial to mild MR in seven. The mean transmitral gradient by intraoperative transesophageal echocardiography was $31 \mathrm{~mm} \mathrm{Hg}$ (range 2 to $6 \mathrm{~mm}$ $\mathrm{Hg}$ ). Postoperatively, seven patients required longer than 24 hours of mechanical ventilator support after mitral reconstruction, but only three patients required longer than 48 hours. There was one late wound infection. No in-hospital deaths occurred, other than the one previously described, and the mean duration of hospitalization after the operation was $9 \pm 4$ days (range 5 to 37 days).

Follow-up was available for all patients. The duration of follow-up in these patients has been 1 to 48 months, with 1- and 2-year survivals of $82 \%$ and $72 \%$. At a mean follow-up of 22 months, all remaining patients are in NYHA class I or II, with a mean postoperative ejection fraction of $26 \%$ and a significant increase over the preoperative ejection fraction in every patient and in the group as a whole. All patients have continued to receive medical therapy for congestive heart failure. Paradoxically, many patients have had their afterload reduction medication increased, because they are now able to tolerate higher doses of angiotensin-converting enzyme inhibitors. All patients have remained in their preoperative rhythm. All patients reported subjective improvement in their functional status after the operation, citing activities routinely performed after the operation for which exertional dyspnea was previously prohibitive. The NYHA failure class significantly fell for every patient individually and from a mean of $3.9 \pm 0.2$ to $1.8 \pm 0.4$ for the entire group. In patients in whom a preoperative comparison was available, peak maximal volume of oxygen use during exercise rose significantly from a mean of 14.5 to $18.6 \mathrm{ml}$ oxygen per kilogram per minute.

Follow-up echocardiography at 1 week, 6 months, and 24 months was available for all patients at those time points. The mean transmitral gradient on follow-up at 1 week was $3 \pm 1 \mathrm{~mm} \mathrm{Hg}$ (range 1 to $5 \mathrm{~mm}$ $\mathrm{Hg}$ ). Matched preoperative and 24-month postoperative measurements of LV size, Doppler-derived flow, and volumes are summarized in Table I. All
Table I. Matched preoperative and 24-month postoperative echocardiographic measurements to compare preoperative and intermediate-term $L V$ end-diastolic volume (EDV), sphericity (diameter) length, $D / L)$, ejection fraction $(E F)$, forward cardiac output $(C O)$, and regurgitant fraction $(R F)$

\begin{tabular}{lccccr}
\hline & $E D V$ & & $E F$ & $C O$ & \\
& $(\mathrm{ml})$ & $D / L$ & $(\%)$ & $(\mathrm{L} / \mathrm{min})$ & \multicolumn{1}{c}{$R F(\%)$} \\
\hline Preop. & $281 \pm 86$ & $0.82 \pm 0.10$ & $17 \pm 3$ & $3.3 \pm 0.9$ & $68 \pm 14$ \\
Postop. & $206 \pm 88$ & $0.74 \pm 0.07$ & $26 \pm 8$ & $5.2 \pm 1.1$ & $15 \pm 14$ \\
Change & $-75 \pm 33$ & $-0.08 \pm 0.07$ & $+9 \pm 5$ & $1.9 \pm 0.9$ & $-53 \pm 18$ \\
$p$ Value & $<0.001$ & 0.005 & 0.008 & 0.001 & $<0.001$ \\
\hline
\end{tabular}

patients had a marked reduction in sphericity, regurgitant volume, and regurgitant fraction and significantly improved LV ejection fraction, end-diastolic volume, and end-systolic volume. Actuarial survival is shown in Fig. 1.

There have been 10 late deaths in this group after mitral valve reconstruction. Two late deaths were related to complications developing after other operative procedures-emergency appendectomy and cholecystectomy. Other deaths have resulted from continued heart failure, suicide, and sudden ventricular arrhythmia.

\section{Discussion}

For patients with dilated cardiomyopathy, mortality is directly related to severity of ventricular systolic dysfunction. ${ }^{11}$ In addition, increased chamber sphericity and, importantly, the presence of $\mathrm{MR}^{12}$ are markers of worsened prognosis. In a review of this type of patients, 1-year mortality has been reported between $54 \%$ and $70 \% .{ }^{13}$ Furthermore, in a study of 130 similar patients awaiting transplantation, 1-year survival was only $46 \%$ and independent predictors of death were low forward stroke volumes, ejection fraction less than $25 \%$, and, again, MR. ${ }^{14}$ The patients in this present study, therefore, represented a high predicted mortality among patients with dilated cardiomyopathy, because they all had severe LV systolic dysfunction, spherical changes in LV geometry, and severe MR.

The pathogenesis of this myopathy-related MR is multifactorial, and its understanding may lead to better surgical approaches. In the absence of organic disease, MR is predominantly thought to occur as a result of progressive dilation of the mitral anulus with subsequent loss of coaptation of the valve leaflets. ${ }^{15}$ In comparisons of patients with dilated cardiomyopathy, with or without MR, those 


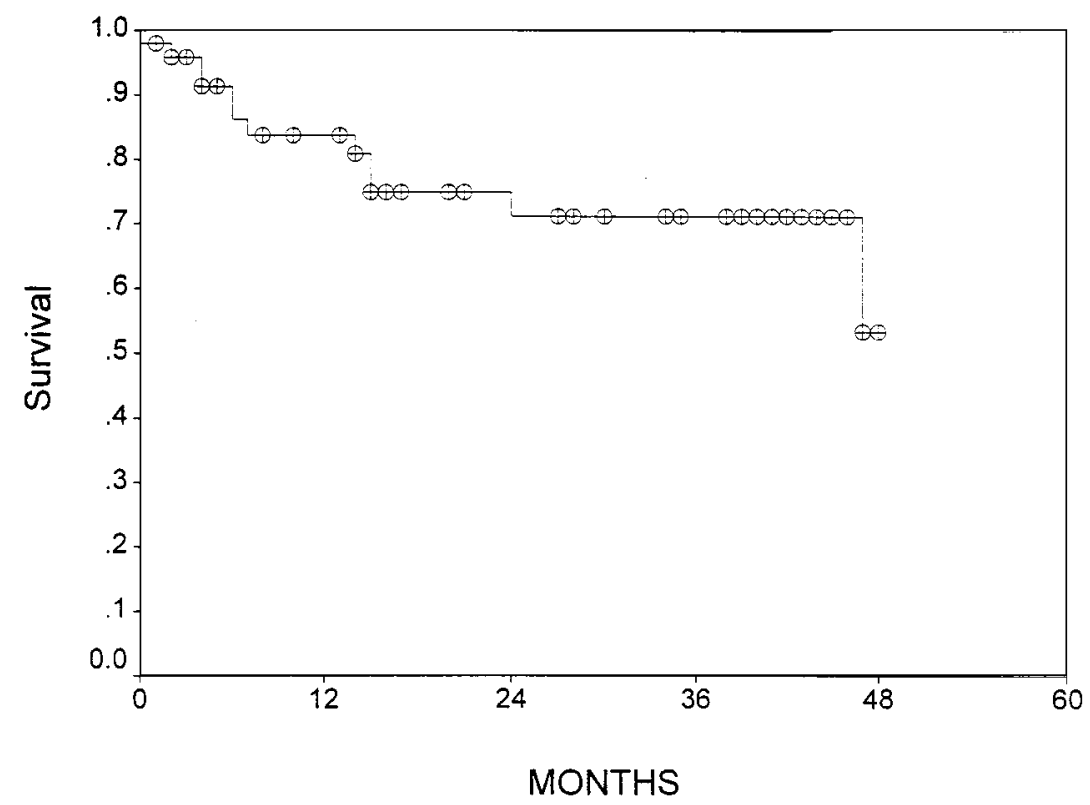

Fig. 1. Actuarial survival after mitral valve reconstruction in cardiomyopathy, as determined by the Kaplan-Meier method.

with MR have significantly greater mitral leaflet orifice surface area and significantly larger dimensions of the mitral valve anulus. ${ }^{8}$ As the size of the mitral valve anulus increases, an increasing amount of redundant mitral leaflet tissue, usually used for coaptation of the leaflets, becomes available to cover the surface area of the mitral valve orifice. A large leaflet area is normally required for coaptation, because mitral valve leaflet area is $2 \frac{1}{2}$ times greater than the area of the mitral valve orifice. ${ }^{16} \mathrm{As}$ more mitral leaflet tissue is used for coverage of the enlarging mitral valve orifice, as the cardiomyopathy progresses, a critical reduction in leaflet tissue available for coaptation is reached, so that coaptation of the mitral leaflets becomes "ineffective" and a central regurgitant jet of functional insufficiency begins to develop. ${ }^{8,17}$ Therefore, the most significant determinant of mitral valve coaptation, leaflet orifice area, and MR is the dimensions of the mitral valve anulus. LV dimension is a less important factor in "functional" MR, because chordal length and angulation or papillary muscle length is not significantly different in patients with idiopathic cardiomyopathy, with or without MR. ${ }^{8}$

However, in ischemic cardiomyopathy, the mechanisms that contribute to MR are more complex and may include "functional" MR through dilation of the mitral valve anulus and "papillary muscle dys- function." Normal function of the mitral valve is dependent on the structural and functional integrity of the subvalvular apparatus. ${ }^{18}$ Papillary muscle dysfunction is actually an unsuccessful coordination of the entire mitral valve apparatus that includes the anulus, leaflets, chordae tendineae, papillary muscles, and the LV wall, rather than simply an isolated disorder of the papillary muscle. ${ }^{19}$ In experimental studies in dogs by Tsakiris and colleagues, ${ }^{20}$ isolated injection of both papillary muscles that induced fibrosis did not, by itself, induce MR. However, if the free wall beneath the papillary muscles is made fibrotic, so that regional asynergy develops, MR develops as a result of restriction of motion of a portion of the leaflets. Gorman and coworkers ${ }^{21}$ reported that ischemic MR appeared to be a multifactorial process that results in significant abnormal deformational changes in the mitral valve anulus. These ischemia-related changes all are thought to result in an insufficient area of coaptation of the mitral leaflet. All of these finding point to a relationship between mitral annular area, lack of coaptation, and the MR seen with myopathy. This relationship may explain why paradoxically an undersized valvular repair can help a muscle problem. Although significant undersizing was used to increase coaptation in all cases, no systolic anterior motion of the anterior leaflet was noted. This sys- 
tolic anterior motion was avoided by widening of the aorto-mitral angle and increased LV size, seen in myopathy.

The surgical management of MR has evolved significantly. Early methods to correct MR simply involved valve replacement with little understanding of the adverse consequences that valve replacement and interruption of anulus-papillary muscle continuity had on short- and long-term LV function. Numerous clinical and experimental studies demonstrate the importance of preserving anulus-papillary muscle continuity of the mitral valve. Lillehei, Levy, and Bonnabeau ${ }^{22}$ first reported on preservation of papillary muscles and chordae tendineae and its benefits on LV function after mitral valve replacement. Along with Rushmer, ${ }^{23}$ who reported a high incidence of postoperative "low cardiac output syndrome" after mitral valve replacement, these investigators subsequently introduced techniques of preservation of the mitral apparatus. Studies by Hansen ${ }^{24}$ Sarris, ${ }^{25}$ and their coworkers, using loadindependent measurements of LV contractility, demonstrate that division of all chordae tendineae was accompanied by a $47 \%$ reduction in maximal LV elastance. Further, the effects of chordal detachment and reattachment on LV function were assessed by load-independent measurements of maximal elastance. When all chordae were detached, a significant decline of LV contractility was seen, which was subsequently restored to baseline levels after the native chordae were reattached by repair of the papillary muscles. As the contributions of the mitral anulus and LV geometry to LV function are further understood, surgeons better appreciate the importance of maintaining chordal, annular, and subvalvular continuity and mitral geometric relationships, which may be even more important in patients with severely compromised LV function.

Because the availability of transplantation as therapy for patients with cardiomyopathy is hindered, there has been recent interest in the altered geometry of LV dysfunction as it relates to cardiomyopathy. This pioneering work has been undertaken by Batista's group ${ }^{3}$ and others and has examined the aberrations in LV mass/volume relationships in patients with cardiomyopathy. Furthermore, surgeons have attempted to renormalize this relationship by LV myoreduction surgery, also called partial LV reduction surgery, reduction myoplasty, or the Batista procedure. This procedure has met with varying degrees of success. However, some survivors appear to be doing well and have been put into
NYHA classes I and II. In Batista's procedure a rough mathematical formula is used to reduce the LV mass/volume ratio to normal. This formula entails a 3:1 ratio of $\mathrm{LV}$ circumference to $\mathrm{LV}$ diameter, so that for a $10 \mathrm{~cm}$ diameter heart to be reduced to a $3 \mathrm{~cm}$ diameter (or by $7 \mathrm{~cm}$ ), the outside circumference of the left ventricle must be reduced by $21 \mathrm{~cm}(3 \times 7)$. This procedure therefore throws away a large amount of good LV mass, as opposed to mitral reconstruction alone, in which no myocardial mass is lost. With mitral valve reconstruction for myopathy, a more normal LV mass/volume ratio is reestablished, with larger LV volumes than have been reported by Batista and others. The average LV volume 24 months after operation in this study was more than $200 \mathrm{ml}$, still quite abnormal, whereas Batista and others have demonstrated an acute reduction of LV volumes to roughly 90 to $100 \mathrm{ml}$ at the time of operation. However, inasmuch as there is no loss of ventricular mass with mitral valve reconstruction, an appropriate mass/volume ratio is still obtained. Importantly, the surgical mortality both at 30 days and at 1 year is much lower in our present series than in any reported series of LV reduction surgery.

Another possible mechanism by which mitral valve reconstruction helps these myopathic hearts may be acute remodeling of the base of the heart from the undersizing of the mitral annular ring. This has been shown by two-dimensional echocardiography immediately in the operating room. This may reestablish an ellipsoid shape to the base of the LV cavity. Additionally, the incidence of sudden death after mitral reconstruction in these patients has not been excessive, perhaps because of the lack of a large ventricular suture line, as in ventricular reduction surgery. Finally, with the Batista procedure, one must differentiate the effect of abolishment of MR alone versus the addition of myoreduction. A recent study denotes that remodeling of LV geometry may be rapid and complete in patients with resultant regurgitant fractions of less than $30 \%$ after correction of MR alone. ${ }^{26}$ Our patients had a mean residual regurgitation fraction of only $16 \%$ after the operation, which could favor rapid LV remodeling. As evidence, late echocardiographic analyses in our patients revealed a decreased sphericity and LV volume after the operation. These patients may be undergoing slow auto-remodeling as opposed to acute Batista remodeling from altering the angulation of the base of the heart, stabilization of the mitral anulus, or LV unloading that has induced a 
more favorable ventricular geometry. This phenomenon appears to occur more readily in the idiopathic hearts than in the end-stage ischemic hearts, which may have less potential for remodeling. Whatever the mechanism, it is interesting to speculate on the long-term effect of these types of LV remodeling operations and whether the effect of unloading the myocyte is permanent. Finally, however, it must be noted that patients in our current series are a carefully selected group who may have more severe MR, slightly better function, smaller LV cavity size, or a higher incidence of chronic illness, with fewer patients receiving inotropic support.

In conclusion, mitral reconstruction by means of annuloplasty effectively corrected MR in these patients with myopathy. Furthermore, despite high risks, there was only one operative death and the actuarial survivals were $82 \%$ and $72 \%$ at 1 and 2 years. Not only was survival improved, but functional status has changed remarkably. Although longer term follow-up with a greater number of patients and perhaps case-matched controls is necessary, we continue to be encouraged by these intermediate results and believe that mitral reconstruction offers a new strategy for patients with end-stage cardiomyopathy.

\section{REFERENCES}

1. Dreyfus GD, Duboc D, Blasco A, et al. Myocardial viability assessment in ischemic cardiomyopathy: benefits of coronary revascularization. Ann Thorac Surg 1994;57:1402-8.

2. Carpentier A, Chachques JC, Acar C, Relland J, Mihaileanu $\mathrm{S}$, Bensasson D, et al. Dynamic cardiomyoplasty at seven years. J Thorac Cardiovasc Surg 1993;106:42-54.

3. Batista RJV, Santos JLV, Takeshita N, Bocchino L, Lima PN, Cunha MA. Partial left ventricular function in end-stage heart disease. J Card Surg 1996;11:96-7.

4. Bolling SF, Deeb GM, Brunsting LA, Bach DS. Early outcome of mitral valve reconstruction in patients with end-stage cardiomyopathy. J Thorac Cardiovasc Surg 1995;4:676-83.

5. Bolling SF. Mitral reconstruction for cardiomyopathy. Nippon Geka Gakkai Zasshi 1995;24:226-7.

6. Bach DS, Bolling SF. Early improvement in congestive heart failure after correction of secondary mitral regurgitation in end-stage cardiomyopathy. Am Heart J 1995;129:1165-70.

7. Bach DS, Bolling SF. Improvement following correction of $2^{\circ}$ mitral insufficiency in end-stage cardiomyopathy with mitral annuloplasty. Am J Cardiol 1996;78:966-9.

8. Boltwood CM, Tei C, Wong M, Shah PM. Quantitative echocardiography of the mitral complex in dilated cardiomyopathy: the mechanism of functional mitral regurgitation. Circulation 1983;68:498-508.

9. Kono T, Sabbah HN, Rosman H, Alam M, Jafri S, Goldstein $\mathrm{S}$. Left ventricular shape is the primary determinant of functional mitral regurgitation in heart failure. J Am Coll Cardiol 1992;20:1594-8.

10. Izumi S, Miyatake K, Beppu S, et al. Mechanism of mitral regurgitation in patients with myocardial infarction: a study using real-time two-dimensional Doppler flow imaging and echocardiography. Circulation 1987;76:777-85.

11. Blondheim DS, Jacobs LE, Kotler MN, Costacurta GA, Parry WR. Dilated cardiomyopathy with mitral regurgitation: decreased survival despite a low frequency of left ventricular thrombus. Am Heart J 1991;122(3 Pt 1):763-71.

12. Juilliere Y, Danchin, Briancon S, Khalife K, Ethevenot G, Balaud A, et al. Dilated cardiomyopathy: long-term follow-up and predictors of survival. Int J Cardiol 1988;21:26977.

13. Anguita M, Arizon JM, Bueno G, Latre JM, Sancho M, Torres F, et al. Clinical and hemodynamic predictors of survival in patients aged less than 65 years with severe congestive heart failure secondary to ischemic or nonischemic dilated cardiomyopathy. Am J Cardiol 1993;72: 413-7.

14. Stevenson LW, Fowler MB, Schroeder JS, Stevenson WG, Dracup KA, Fond V. Poor survival of patients with idiopathic cardiomyopathy considered too well for transplantation. Am J Med 1987;83:871-6.

15. Fischl SJ, Gorlin R, Herman MV. Cardiac shape and function in mitral valve disease: physiologic and clinical implications. Am J Cardiol 1977;39:170.

16. Hendren WG, Nemec JJ, Lytle BW, Loop FD, Taylor PC, Stewart RW, et al. Mitral valve repair for ischemic mitral insufficiency. Ann Thorac Surg 1991;52:1246-52.

17. Chandraratna PAN, Aronow WS. Mitral valve ring in normal vs dilated left ventricle: cross-sectional echocardiographic study. Chest 1981;79:151.

18. Perloff JK, Roberts WC. The mitral apparatus: functional anatomy of mitral regurgitation. Circulation 1972;46:227-39.

19. Kay GL, Kay JH, Zubiate P, Yokoyama T, Mendez M. Mitral valve repair for mitral regurgitation secondary to coronary artery disease. Circulation 1986;74(Suppl):I88-98.

20. Tsakiris AG, Rastelli GC, Amorim D, Titus JL, Wood EH. Effect of experimental papillary muscle damage on mitral valve closure in intact anesthetized dogs. Mayo Clin Proc 1970;45:275-85.

21. Gorman RC, McCaughan JS, Ratcliffe MB, Gupta KB, Steicher JT, Ferrari VA, et al. Pathogenesis of acute ischemic mitral regurgitation in three dimensions. J Thorac Cardiovasc Surg 1995;109:684-93.

22. Lillehei CW, Levy MJ, Bonnabeau RC. Mitral valve replacement with preservation of the papillary muscles and the chordae tendineae. J Thorac Cardiovasc Surg 1964;47:53243.

23. Rushmer R. Initial phase of ventricular asystole: asynchronous contraction. Am J Physiol 1956;188:187-94.

24. Hansen DE, Cahill PD, DeCampli WM, et al: Valvularventricular interaction: importance of the mitral apparatus in canine left ventricular systolic performance. Circulation 1986;73:1310-20.

25. Sarris GE, Cahill PD, Hansen DE, et al. Restoration of left ventricular systolic performance after reattachment of the mitral chordae tendineae: the importance of valvular-ventricular interaction. J Thorac Cardiovasc Surg 1988;95:969-79.

26. Nagatsu M, Ishihara K, Zile MR, et al. The effects of complete versus incomplete mitral valve repair in experimental mitral regurgitation. J Thorac Cardiovasc Surg 1994;107: 416-23. 


\section{Discussion}

Dr. Kent W. Jones (Salt Lake City, Utah). When the group from the University of Michigan presented their early outcome of mitral valve reconstruction in this group of patients at the Western Thoracic Surgical Association meeting in Squaw Valley in 1994, their results were received with astonishment. Discussants of the paper prefaced their remarks by such statements as:

"It would not have been intuitively obvious to me that these patients would have benefited from this operation." "These are truly surprising results." "I am flabbergasted." "These results are absolutely phenomenal." Now, 3 years later, we remain very impressed by these results.

The series now comprises 47 consecutive nonrandomized patients with end-stage cardiomyopathy and severe MR who were treated by mitral valve reconstruction using a flexible annuloplasty ring. Some of the most impressive and important facts resulting from this study are as follows:

1. The mean time from documentation of the cardiomyopathy to operation was 4 years.

2. Only one operative death and no hospital deaths occurred, and the mean hospital stay was only 9 days.

3. The 1-year actuarial survival was $82 \%$ and the 2-year actuarial survival was $72 \%$.

4. Postoperatively, all patients were in NYHA class I or II and the mean left ventricular ejection fraction was $26 \%$.

5. Nine patients were accepted for the operation, but declined. Seven of these nine were dead within 8 months.

This procedure represents one of a spectrum of new modalities that have evolved in the treatment of patients with end-stage heart disease who would otherwise be candidates for cardiac transplantation. However, given the limitations imposed on transplantation because of organ shortage, age, and its restricted applicability in patients with concurrent disease, the results presented here certainly provide another potential avenue of treatment.

I have three questions: First, since this group of patients has survived an average of 4 years after initial documentation of their cardiomyopathy, do they represent a select group? Most patients with end-stage heart disease have died within this time frame.

Dr. Bolling. These are very carefully selected patients; the vast majority of these patients were operated upon on an outpatient basis. If we could do anything to get them out of the hospital, off inotropic support, see them in the clinic, and bring them back when they were in more stable condition, we did that. As opposed to the Batista series and the Cleveland Clinic series, I think we were operating on less acutely ill patients. Only two patients in this series were receiving inotropic support at the time we operated on them.

Dr. Jones. Second, inasmuch as MR is usually a late complication of cardiomyopathy and is a poor prognostic sign, what gave your group the impetus to undertake this study?

Dr. Bolling. We saw patients who were on our transplant list in whom MR developed late in the their course, and then they would die before we could get a donor organ for them. We asked ourselves, "What could we do for these patients to tide them over to transplantation?"

Dr. Jones. Third, this study, like the initial early outcome study, represents consecutive, nonrandomized patients. Would not a study using case-matched controls offer more meaningful information in this group of patients?

Dr. Bolling. I think that is an absolutely great idea. A multiinstitutional, case-matched, randomized study of this operation versus mitral reconstruction plus myoreduction, the so-called Batista procedure, would be indicated in this group of patients. We do not have a control group, and we should have a placebo or randomized approach to these patients. We had planned to operate on these patients, and then for various reasons (they became sick and died, or they went away and we did not hear back from them) we did not operate. They are not control patients; they are not randomized; I do not want to put weight on those patients. However, medical therapy for cardiomyopathy yields pretty good results in this group of patients. Routine death rates on a heart transplant waiting list are no longer $100 \%$ at 1 year with inhibition of angiotensinconverting enzyme. However, mortality is very high in those specific patients with $4+$ MR refractory to medical therapy. These patients were similar to patients we would have operated on. That is all we are implying.

Dr. David A. Fullerton (Winnetka, Ill.). Dr. Bolling, since the last time you and I spoke about this, we have operated on several patients who I think would fit into your group. The preoperative ejection fraction has been around $20 \%$ in all, and 2 weeks after the operation the ejection fraction was about $26 \%$ or $27 \%$ according to multiple gated acquisition scan.

According to what has been written about doing coronary bypass surgery in patients who have very poor ejection fractions, those patients who had coronary bypass had a better long-term survival if they had an electrophysiologic study after the operation. I extrapolated from those data and asked our electrophysiology group to do studies in patients getting mitral repairs. What we found was that most of this group had a positive electrophysiologic study and received an automatic implantable cardioverter-defibrillator. Have you given any thought to that, and might that strategy offer an enhanced long-term survival for this group of patients?

Dr. Bolling. That is a good question, Dr. Fullerton. We have looked at the same issue in these patients-whether over the long term we should add an automatic implantable cardioverter-defibrillator or whether all these patients should be receiving amiodarone. There is an incidence of late sudden death, both in the idiopathic group and in the ischemic group, as is true in all patients with cardiomyopathy, and that is certainly an interesting suggestion.

Dr. Sulaiman B. Hasan (Karachi, Pakistan). I have two questions: What was the incidence of significant pulmonary hypertension and tricuspid regurgitation, and did you select on the basis of that?

Dr. Bolling. We had wide ranges of pulmonary hypertension from some patients having supersystemic pulmonary artery pressures at the time of operation. The 
incidence of significant tricuspid regurgitation is about $25 \%$ in this series, and about $25 \%$ of those patients underwent tricuspid annuloplasties. That was usually an intraoperative decision.

Dr. Hasan. Did you see any difference in the morbidity in the patients who had higher pulmonary artery pressures?

\section{Dr. Bolling. No.}

Dr. Hasan. What kind of annuloplasty ring do you use?

Dr. Bolling. We used both a flexible and a semiflexible ring. One is a Physio ring (Baxter Healthcare Corp., Irvine, Calif.) and the other is the Duran ring (Medtronic, Inc., Minneapolis, Minn.). We are actually trying to randomize between a totally flexible ring and a semiflexible ring in terms of patient outcome, but we do not have enough patients to say anything about that yet.

Dr. Jay I. LaBourene (Belvedere, Calif.). I was involved in the early part of this study as a resident. At that time we were measuring the intertrigonal distance to determine ring size. I have operated on about a dozen patients similar to these using the intertrigonal technique with very good results. I am curious about the fact that you are now downsizing the anulus much more than we used to. What stimulated you to do that? Are you finding geometric or clinical advantages in doing so?

Dr. Bolling. As you know, we would downsize one size or so to try to eliminate MR. However, as we followed up these patients with echocardiography, we noted that we were abolishing the MR, the 2-year data were excellent, and the base of the heart was being reduced. The smaller we made the anulus, the more the base of the heart came in and the more ellipsoid the long radius of the heart became from base to apex. We thought not only were we going to abolish MR, but we were going to acutely remodel the heart to an ellipse. The long-axis radius was going to have more curvature and the radius was going to change. We started downsizing more and more. Now we are basically putting in the smallest rings that we can, and we have not seen mitral stenosis clinically in any patient.

Dr. Robert M. Kass (Los Angeles, Calif.). I am curious about the causes of death in the patients who died in the follow-up period. Also, it appears that there is going to be a significant fall-off as you monitor the patients. Are these patients still maintained on your transplant list?

Dr. Bolling. The causes of death are listed in the article. The biggest cause of death was continued heart failure. We are looking at molecular markers. We would like to see norepinephrine levels and other indicators that would tell us that this patient will never get better. Two of our patients have later required elective general surgery operations, a cholecystectomy and then an emergency appendectomy, and they both died. One woman committed suicide. I think there probably will not be a sustained change in the myocytes. Whether these myocytes are permanently damaged and whether left ventricular function can recover permanently are matters of controversy. The numbers we have at 3 and 4 years are not big enough to allow us to say anything about long-term results, although the Kaplan-Meier curve does fall off at 36 and 48 months. 